\title{
Vulnerability of Zigbee to Impulsive Noise in Electricity Substations
}

\author{
S A Bhatti, Qingshan Shan ${ }^{2}$, R Atkinson ${ }^{1}$, M Vieira, ${ }^{3,1}$ and I A Glover ${ }^{1}$ \\ ${ }^{I}$ Department of Electronic \& Electrical Engineering, University of Strathclyde \\ 204 George Street, Glasgow G1 1XW, UK \\ Email: shahzad.bhatti@eee.strath.ac.uk \\ ${ }^{2}$ MRC Institute of Hearing Research, University Park, Nottingham, NG7 2RD, UK \\ ${ }^{3}$ Department of Electrical Engineering, Universidade Federal de Campina Grande, Brazil.
}

\begin{abstract}
The vulnerability of Zigbee technology to noise in an electricity substation environment is assessed. Substation noise obtained from a measurement campaign is modelled as a Symmetric $\alpha$-Stable process. The parameters of the model are estimated from the measurements and the resulting model is used to investigate the likely BER performance of Zigbee technology deployed in a substation.
\end{abstract}

\section{Introduction}

The use of wireless sensor networks (WSNs) for industrial automation and monitoring has several potential benefits over wired systems including convenience of deployment, ease of reconfiguration and reduced costs. There have been significant recent developments in terms of WSN standardization. The HART Communication Foundation and International Society of Automation (ISA) are particularly influential in the field of wireless industrial automation systems. Wireless HART is a standard that defines a protocol stack that can employ any short range wireless technologies (WLAN, Bluetooth, Zigbee) at its physical layer. Many companies in the health, oil exploration and other sectors have recently adopted Wireless HART. Its use in electricity supply industry, however, is limited, possibly because of a perception the reliable operation will be at risk due to short, but intense, field transients extending into the RF and microwave spectrum during faults and/or switching events. Electricity substations contain transformers, circuit breakers, isolators, cables, voltage regulators, and other equipment for control and protection. Both partial and full discharges can occur within, and across, any degraded insulation forming part of these items of plant. These discharges generate rapid changes in current and thus lead to the radiation of electromagnetic noise typically consisting of a quasi-random train of short (nanosecond) impulses. Corona discharge is one form of partial discharge which occurs when the potential gradient in the gas (usually air) around a charged object (which may or may not be a conductor) exceeds the breakdown threshold. Power system switching events and fault transients also give rise to the radiation of unwanted impulsive noise that may interfere with the reliability or performance of wireless receivers generally and WSNs in particular.

Whilst the assumption of additive white Gaussian noise (AWGN) greatly simplifies the design and analysis of receiver structures this assumption may not be appropriate for many environments including the power systems environment where impulsive noise may dominate.

The work reported here addresses the performance assessment of the Zigbee receiver when deployed in the particular noise environment of an electricity transmission substation (ETS). The paper is divided into three sections. Section 2 addresses the physical layer of Zigbee transceiver (IEEE 802.15.4) and its simulation. Section 3 describes the modeling of impulsive noise using a symmetric alpha stable $(\mathrm{S} \alpha \mathrm{S})$ process. Section 4 comments on the results and draws some conclusions.

\section{Zigbee Technology}

ZigBee is a standard for low power and low data-rate wireless communications. It has a wide range of, applications in home and industrial automation, automation. The application of specific interest here is in the substation segment of smart grids. It was developed by the ZigBee Alliance and its medium access control (MAC) and physical (PHY) layers are based on the IEEE 802.15.4 standard. The standard defines an air interface employing $250 \mathrm{kbit} / \mathrm{s}$, 32-chip, direct sequence spread spectrum (DSSS) and offset quadrature phase shift keying (OQPSK) 
operating in the unlicensed Industrial, Scientific and Medical (ISM) frequency bands. From a total of 27 frequency channels, one lies in the $868 \mathrm{MHz}$ band, ten in the $915 \mathrm{MHz}$ band and 16 in the $2.45 \mathrm{GHz}$ band. Figs. 1 and 2 show Simulink block diagrams of the simplified PHY layer of Zigbee transmitter and receiver for the $868 / 915 \mathrm{MHz}$ and $2.45 \mathrm{GHz}$ bands.

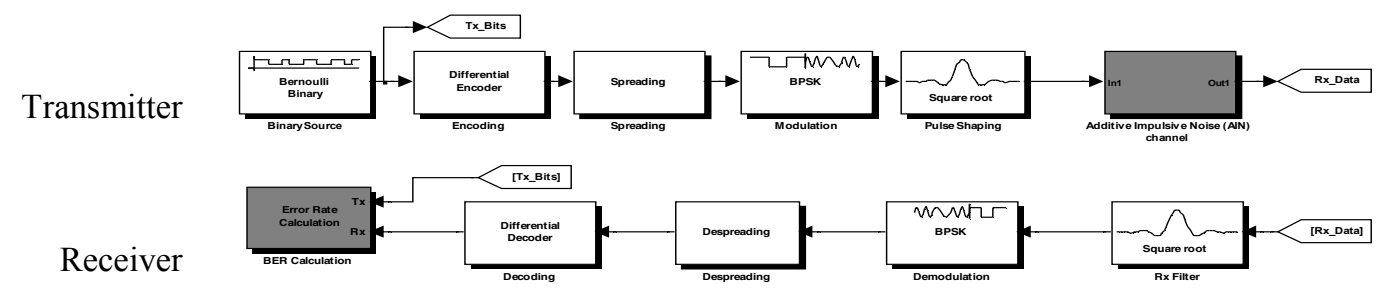

Fig. 1 Simulink block diagram of physical layer of Zigbee transceiver for 868/915 MHz bands

Transmitter

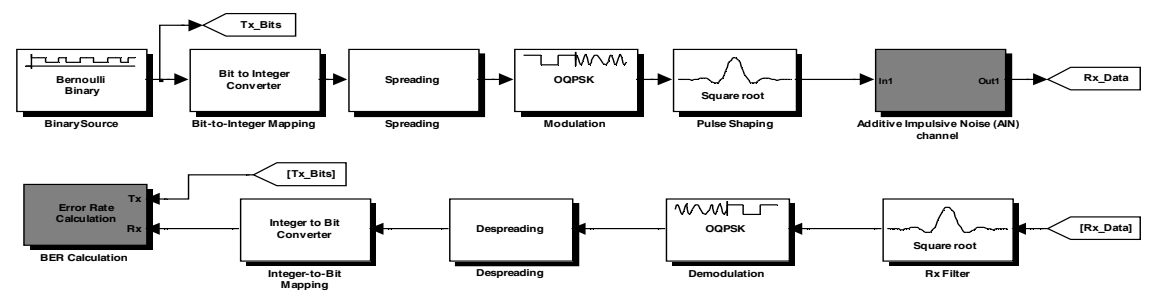

Fig. 2 Simulink block diagram of physical layer of Zigbee transceiver for $2.45 \mathrm{GHz}$ band

\section{Noise Model}

Non-Gaussian impulsive models can be divided into two main categories: empirical and statistical-physical.

Empirical models are based on the fitting of a mathematical expression to experimental data without consideration of the physical noise processes. These models are simple but can only be employed for the particular situation, applying to the data from which they are derived. In this sense they are non-transportable. Gaussian mixture and the hyperbolic distribution models are the most commonly used empirical models [1-2].

Statistical-physical models give at least partial consideration to the underlying physical noise processes. Among statistical-physical models of impulsive noise, Middleton's approach [3] has been very widely adopted. This classifies impulsive noise into one of three types based on the relative bandwidth of the noise and receiver.

The Middleton Class-A model assumes receiver bandwidth is wide compared to the noise bandwidth. In this case the noise does not produce any noticeable transients in the receiver. The Class-B model assumes receiver bandwidth is narrow compared to the noise bandwidth. In this case the noise produces strong transients in the receiver. The Class-C model is a mixture (sum) of Class-A and Class-B models. These models have been shown to provide a quantitative and realistic description of many man-made, and natural, radio noise processes.

The short duration of the impulses generated by partial discharge processes generally make the Class-B model the most appropriate for the power system environment. Class-B noise has seven parameters, however, and is mathematically complex. An approximation to the Class-B model proposed by Nikias and Shao represents impulsive noise using the symmetric $\alpha$-stable (S $\alpha \mathrm{S}$ ) distributions [4]. The characteristic function of an $\mathrm{S} \alpha \mathrm{S}$ process has the form:

$$
f(w)=\left.e^{j \delta w-\gamma|w|}\right|^{\alpha}
$$

A general closed-form expression for its density function does not exist except for two special cases. (The special cases are for $\alpha=2$ which represent Gaussian distributions and for $\alpha=1$ which represent Cauchy distributions). Its density function can be expanded, however, into convergent series using a power series expansion 
[4]. The $\mathrm{S} \alpha \mathrm{S}$ distribution is completely determined by three parameters; $\alpha$ (the characteristic exponent), $\gamma$ (the dispersion parameter) and $\delta$ (the location parameter). The shape parameter defines the 'thickness' of the tails of the $\mathrm{S} \alpha \mathrm{S}$ pdf, i.e. it controls the decay gradient of the pdf tails. For smaller values of $\alpha$ the tails decay slowly corresponding to a highly impulsive process. For values of $\alpha$ close to 2, the tail thickness is close to that of the Gaussian distribution. (For $\alpha=2$ the $\mathrm{S} \alpha \mathrm{S}$ distribution reduces to Gaussian.) For $\alpha=1$ the $\mathrm{S} \alpha \mathrm{S}$ distribution reduces to Cauchy. The dispersion parameter controls the spread of the distribution around its location. It can have any positive value and is similar in function to variance. (For the Gaussian case of $\alpha=2$ the dispersion is equal to half

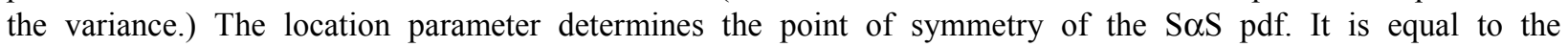
distribution's mean when $1 \leq \alpha \leq 2$. Fig. 3 illustrates the $\mathrm{S} \alpha \mathrm{S}$ density curves for different values of $\alpha$. For small positive values of $\alpha$ the curves have cusped peaks and the pdf tails decay slower than in the Gaussian case $(\alpha=2)$.

The parameters of an $\mathrm{S} \alpha \mathrm{S}$ noise model have been estimated from time-series data recorded in the control room of a 400/275/132 kV air-insulated substation in Strathaven, UK. Details of the data recording system, the measurement campaign and data pre-processing are given in [6,7]. The parameter fitting algorithm uses an estimator described in [5]. It estimates all three parameters, requires only relatively simple computations and is therefore particularly fast. Fig. 4 shows the amplitude probability densities of measured data, best-fit Gaussian distribution and best-fit $\mathrm{S} \alpha \mathrm{S}$ (with parameter estimated from measured data). Fig. 4 demonstrates the suitability of this noise model for the electricity substation environment.

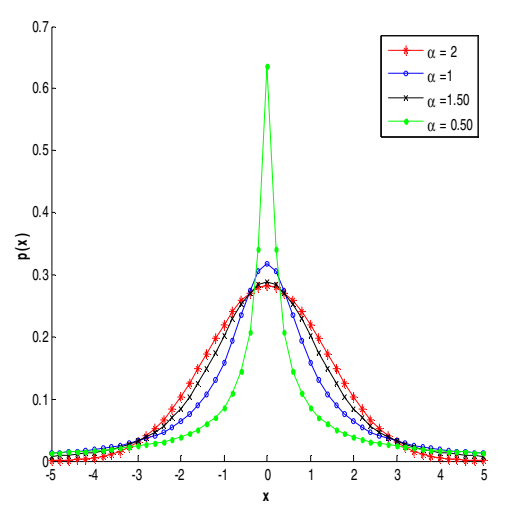

Fig. $3 \mathrm{~S} \alpha \mathrm{S}$ pdfs for different value of $\alpha$

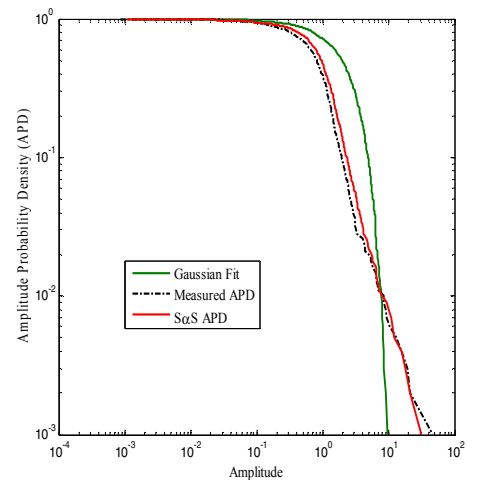

Fig. 4 Amplitude probability densities (APDs) calculated from the recorded data, best-fit $\mathrm{S} \alpha \mathrm{S}(\delta=-0.791, \alpha=1.40$ and $\gamma=0.3079)$ and bestfit Gaussian distributions.

\section{Simulation Results and Discussion}

The physical layer simulation of a Zigbee transceiver (IEEE 802.15.4) described in section 2 has been validated in the presence of AWGN against the theoretically expected BER performance curves. Fig. 5 shows the validation results for both $868 \mathrm{MHz}$ BPSK and $2.45 \mathrm{GHz}$ OQPSK modes. The agreement between simulated results and theory is good evidence that the simulations are accurate. The substation noise environment is modeled using the $\mathrm{S} \alpha \mathrm{S}$ process with parameters computed from the data secured during a measurement campaign.

The $\mathrm{S} \alpha \mathrm{S}$ distribution does not have finite second order moments and all $\mathrm{S} \alpha \mathrm{S}$ signal processing is based on fractional lower order moments (FLOM) [4]. The use of a traditional signal-to-noise ratio (SNR) is therefore not appropriate. BER performance has therefore been characterized as a function of dispersion $(\gamma)$.

The performance of the Zigbee receiver in the substation noise environment is shown in Fig. 6 for three specific values of $\alpha .1$ (corresponding to a Cauchy distribution), 1.40 (corresponding to the noise recorded in the substation) and 2 (corresponding to a Gaussian distribution). For $\gamma<0.3$ the performance degradation with respect to the Gaussian case is negligible. For $\gamma>0.3$, however, the performance degradation is significant. Larger values of $\gamma$ denote a greater dominance of impulsive noise power over Gaussian noise power. The Cauchy distribution represents noise that is more impulsive noise than that recorded in the substation. Hence 

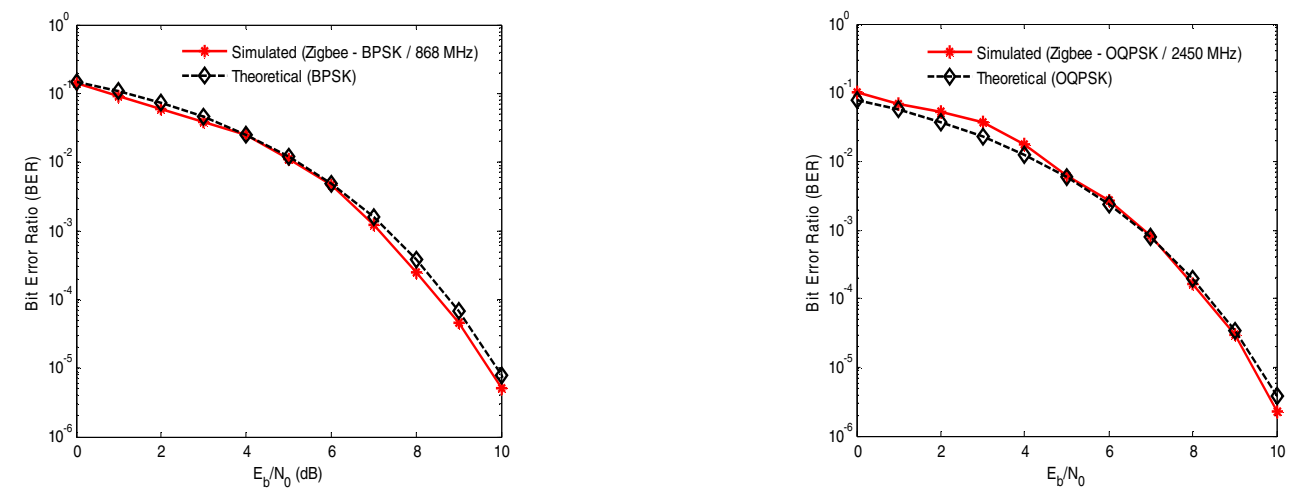

Fig. 5 Validation of the Zigbee simulation for (a) the $868 / 915 \mathrm{MHz}$ bands and (b) the $2.4 \mathrm{GHz}$ band.
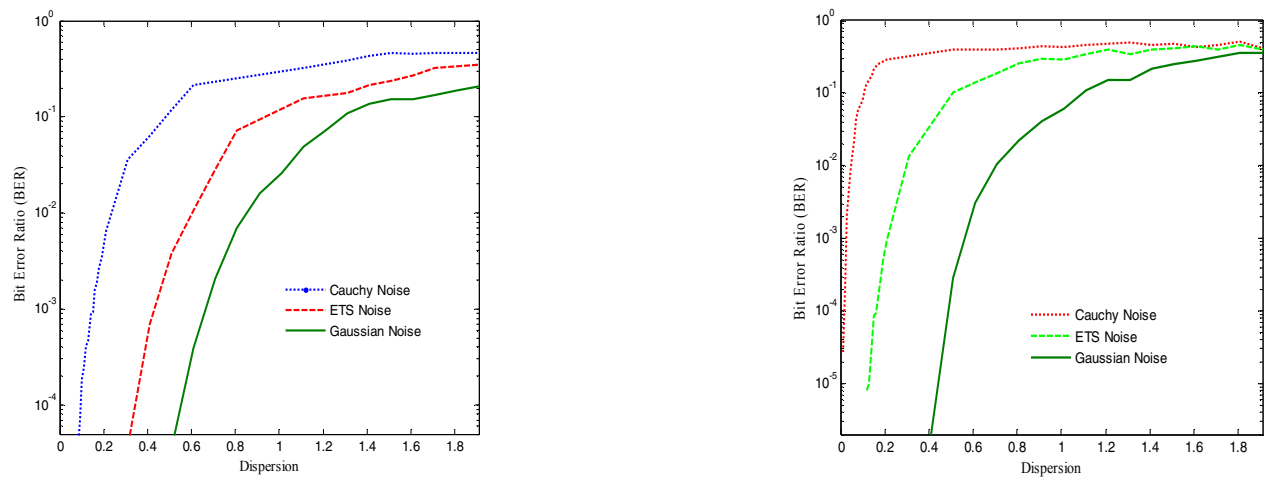

Fig. 6 Simulated performance of Zigbee in the presence of Cauchy $(\alpha=1)$, measured $(\alpha=1.40)$ and Gaussian $(\alpha=2)$ noise for (a) $868 / 915$ bands and (b) $2.4 \mathrm{GHz}$ band.

\section{Acknowledgments}

The authors thank the UK Engineering and Physical Sciences Research Council (EPSRC) and Higher Education Commission of Pakistan (HEC) for financial support of this work.

\section{References}

[1] E. J. Wegman, S. C. Schwartz, and J. B. Thomas, Topics in non-Gaussian signal processing. New York: Springer-Verlag, 1989.

[2] P. Mertz, "Model of Impulsive Noise for Data Transmission," Communications Systems, IRE Transactions on, vol. 9, pp. 130-137, 1961.

[3] D. Middleton, "Non-Gaussian noise models in signal processing for telecommunications: New methods and results for class A and class B noise models," Ieee Transactions on Information Theory, vol. 45, pp. 1129$1149,1999$.

[4] M. Shao and C. L. Nikias, "Signal processing with fractional lower order moments: stable processes and their applications," Proceedings of the IEEE, vol. 81, pp. 986-1010, 1993.

[5] G. A. Tsihrintzis and C. L. Nikias, "Fast estimation of the parameters of alpha-stable impulsive interference," IEEE Transactions on Signal Processing, vol. 44, pp. 1492-1503, 1996.

[6] Q. Shan, I. A. Glover, R. Rutherford, S. A. Bhatti, R. Atkinson, I. E. Portugues, and P. J. Moore, "Detection of ultra-wide-band impulsive noise in a $400 \mathrm{kV}$ air insulated electricity substation," in Electricity Distribution - Part 1, 2009. CIRED 2009. 20th International Conference and Exhibition on, 2009, pp. 1-4.

[7] Q. Shan, S. A. Bhatti, I. A. Glover, R. Atkinson, P. J. Moore, I. E. Portugues, and R. Rutherford, "Characteristics of impulsive noise in electricity substations," in 17th European Signal Processing Conference (EUSIPCO 2009), Glasgow, UK, 2009, pp. 2136-2140. 